\title{
Raman microscopic studies of polymer surfaces and interfaces
}

\author{
C. Sammon ${ }^{1 *}$, C. Mura ${ }^{1,2}$, P. Eaton ${ }^{1,3}$ and J. Yarwood ${ }^{1}$ \\ ${ }^{1}$ Sheffield Hallam University, Materials Research Institute, Howard Street, Sheffield S1 1WB, United Kingdom \\ ${ }^{2} S$ G Magnets Ltd, Tesla House, 85 Ferry Lane, Rainham, Essex RM13 9YH, United Kingdom \\ ${ }^{3}$ University of Portsmouth, School of Pharmacy and Biomedical Sciences, St Michael's Building, White Swan Road, Portsmouth, \\ Hampshire PO1 2DT, United Kingdom
}

We describe three examples of how innovative confocal Raman microscopy can be employed to address questions of commercial importance for polymer-polymer or polymer-substrate interfaces.

aman microscopy is rapidly becoming the technique of choice for the analysis of polymer surfaces and interfaces. The short (visible or UV) source wavelength, combined with high throughput spectrometers $[1,2]$ and very sensitive (CCD) two dimensional detectors provide an analytical tool with molecular discrimination and micron scale mapping capability. The confocal capability of modern Raman microscopes (CRM) gives additional depth-profiling information [2,3,] (without sectioning) for materials "transparent" to the source laser. There are now a wide range of potential applications of such a system and therefore an increasing range of commercial instruments on the market [4]. We outline here just three examples of how this technique can be employed to enhance the quality of materials characterisation

Commercial PET "film" (such as that used for overhead projector slides and other products) is a co-extended biaxially oriented laminate comprising a layer of PET (Fig. 1a and $1 \mathrm{~b}$ ) and PET containing an isophthalate comonomer to reduce crystallinity (Fig. 1c). Since the Raman bandwidth of the " $v(\mathrm{C}=\mathrm{O})$ " mode of PET is sensitive to crystallinity $[5,6]$ it is possible to quantify the crystallinity gradient as shown in figure $2 \mathrm{a}$. Comparison of CRM data with the result obtained by microtoming (to provide "lateral" measurements across the interface) shows good agreement (Fig. 2b) and underpins the validity of the confocal approach within the limit of a $2 \mu \mathrm{m}^{3}$ spatial resolution.

An important issue around the incorporation of fungicides into paints and other polymer-containing coatings and consumer products is the potential loss of "active" in the ambient environment [7,8]. Figure 3 shows how CRM may be employed to map and depth profile the fungicide (PA3) content of a plasticised PVC film using the ratio of band intensities shown in figure $3 \mathrm{~b}$. The leaching process (water at

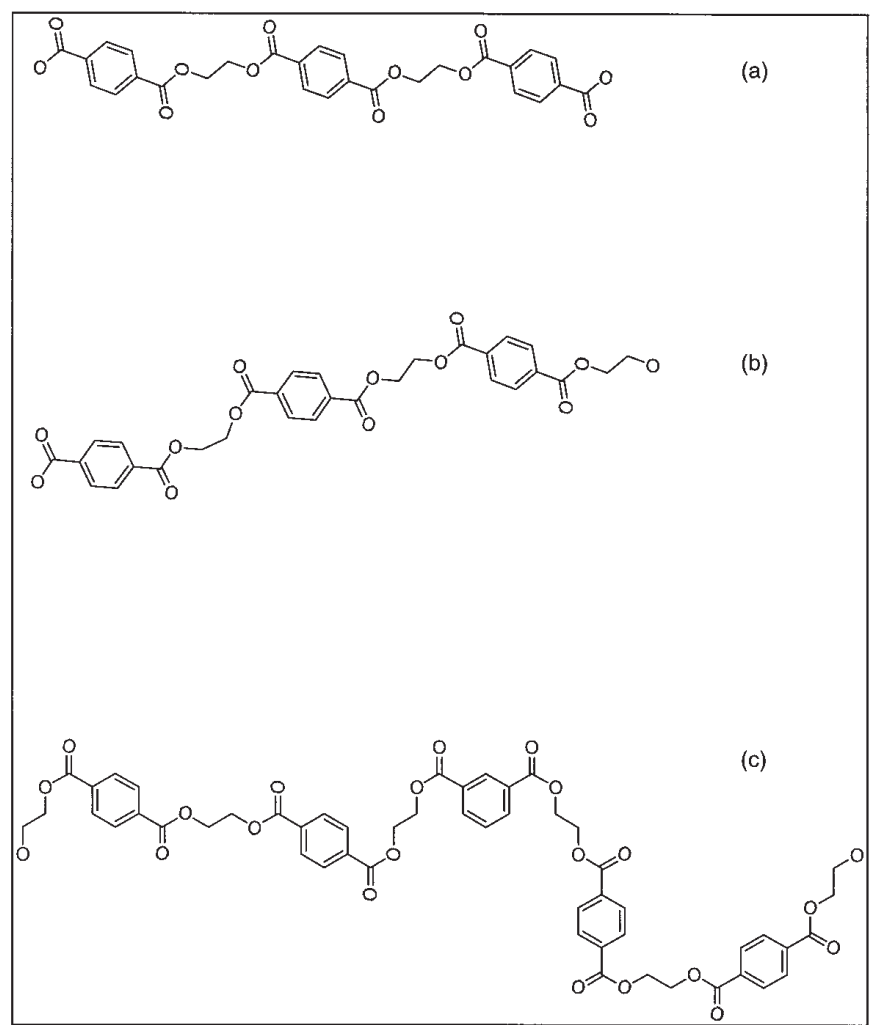

Figure 1. (a) E47 PET in an all "trans" crystalline formation; (b) E47 Pet in a mixed "trans" and "gauche" amorphous conformation; (c) E99 PET containing a fixed percentage of isophthalic acid comonomer.

$25{ }^{\circ} \mathrm{C}$ for several hours) clearly leads to a loss of fungicide both from the surface and from the bulk (Fig. 3c) of the film. Such a loss of toxic material to the ambient has potential environmental impact and is therefore of significant commercial importance.

The distribution of silane primer (such as Y9669 - see figure 4) across a polymer/glass laminate (Fig. 4a) and, in particular, accumulation at the polymer/glass interface is thought $[9,10]$ to be a controlling factor for the adhesion mechanism in such materials. The relative amount of silane at a given depth into the polymeric material may be assessed 


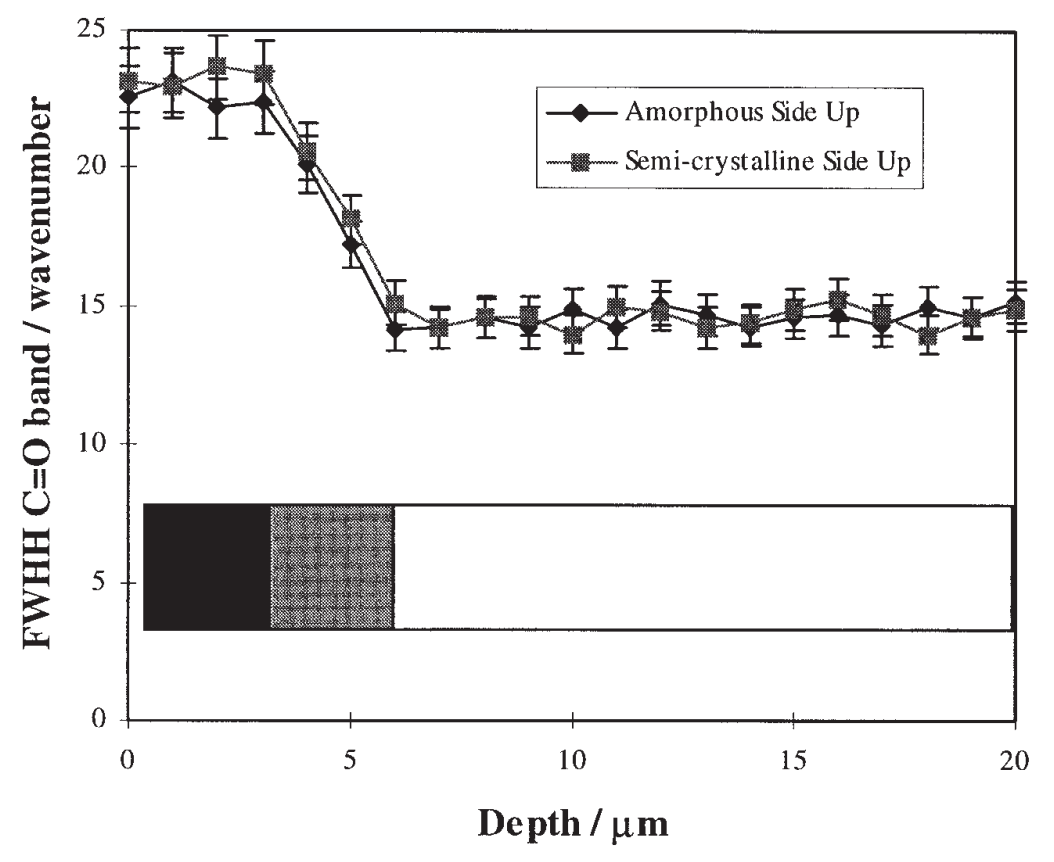

a

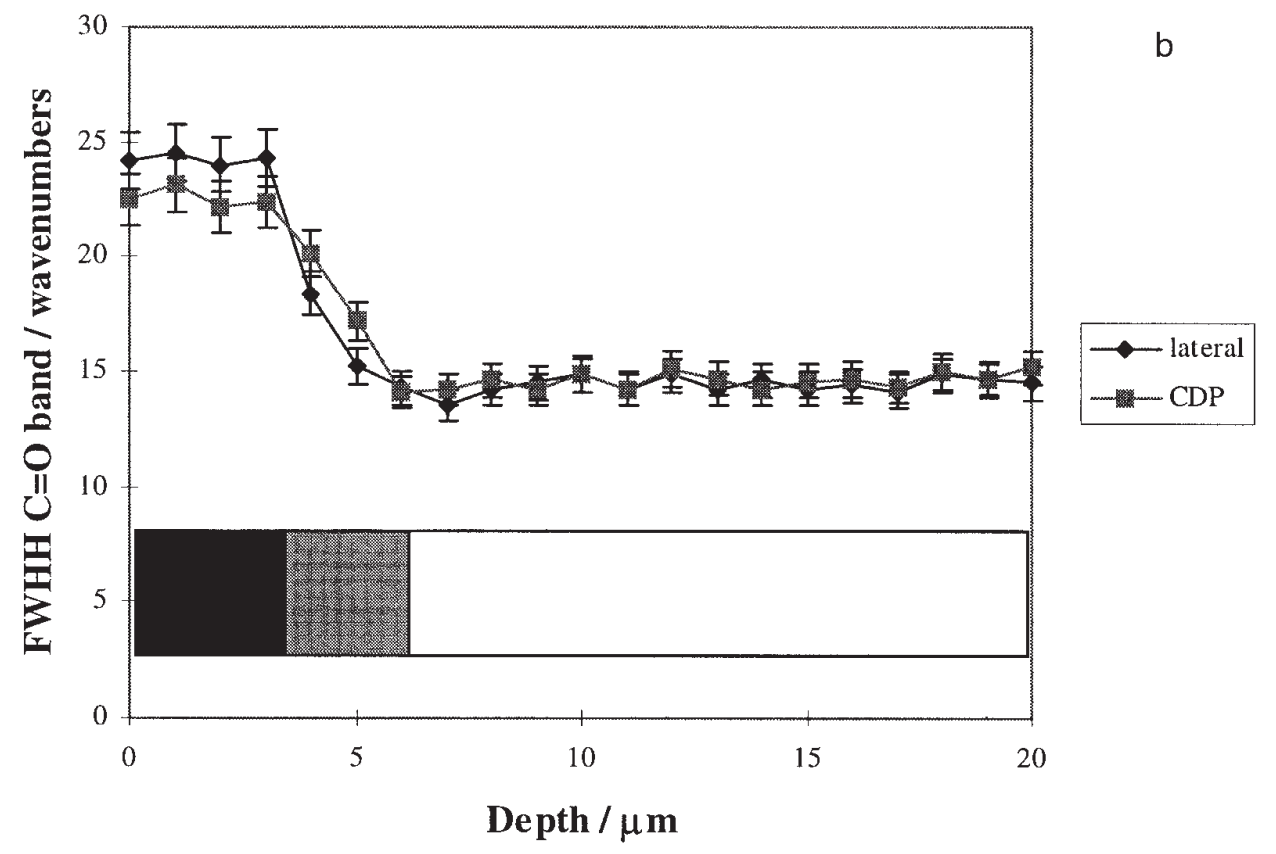

Figure 2. (a) The $v(C=0$ ) band width as a function of depth (in confocal mode) for a $30 \mu \mathrm{m}$ PET laminate; (b) Comparison of the interfacial gradients measured for a $20 \mu \mathrm{m}$ PET laminate for the two depth profile experiments. 
Fluorofolpet (PA3)

(a)<smiles>O=C1c2ccccc2C(=O)N1SC(F)(Cl)Cl</smiles>

(b)

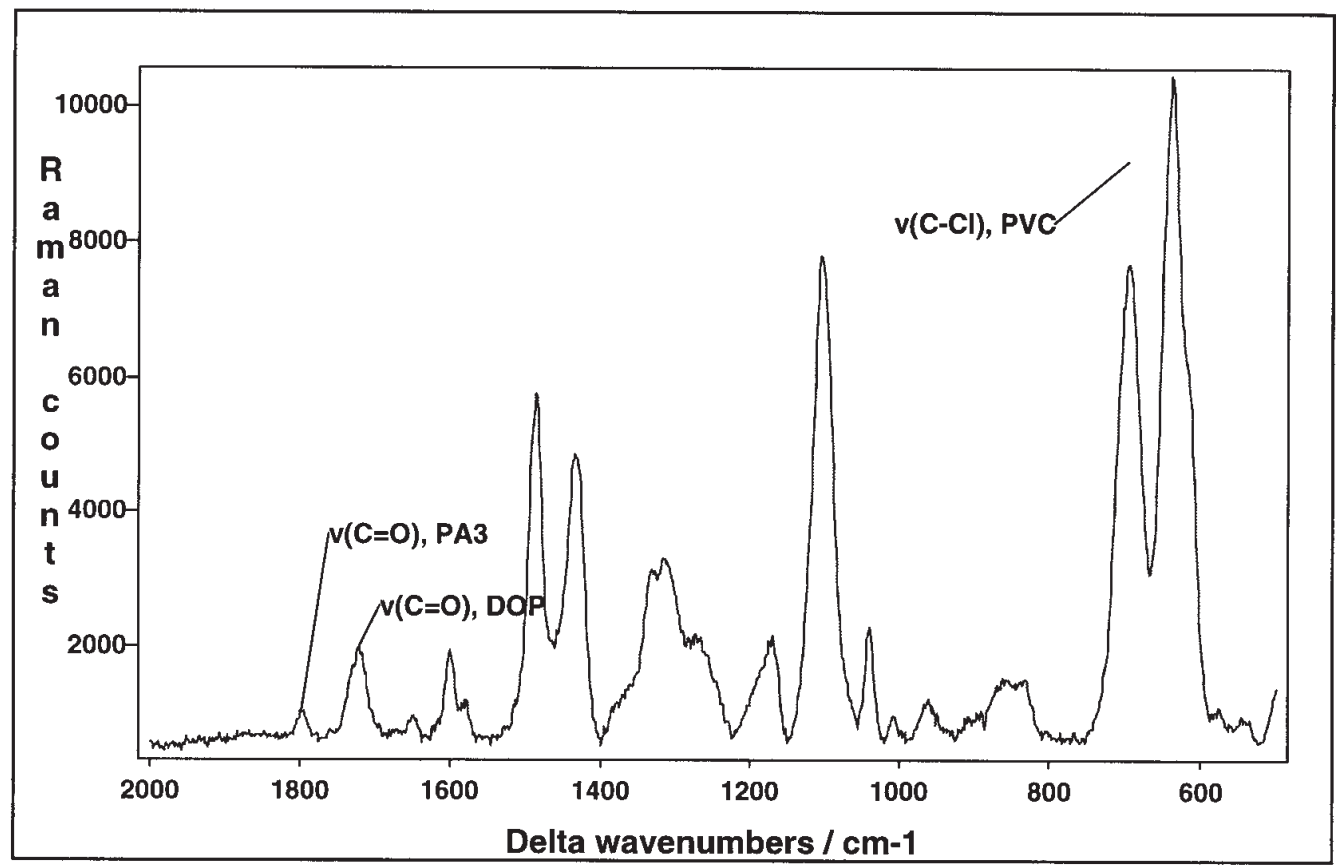

(c)

Depth Profile

$25 \% \mathrm{DOP} / 5 \% \mathrm{PA} / \mathrm{PNC}$

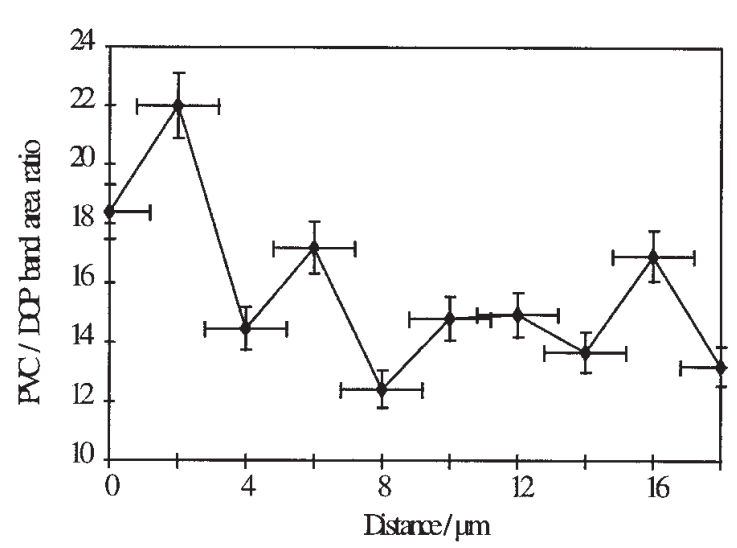

X-Y Map

Distribution of fluorfolpet

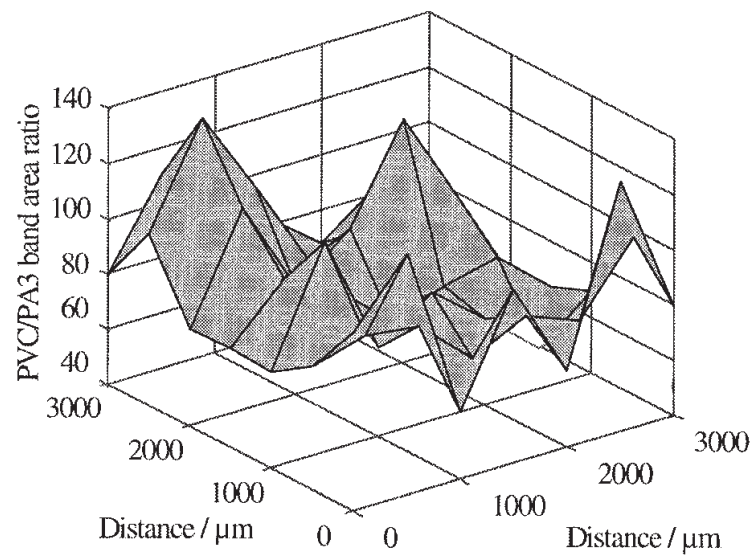

Figure 3. (a) The structure of Fluorofolpet (PA3); (b) the Raman spectrum of biocide (PA3) and plasticiser (DOP) in a PVC matrix, (c) map and depth profile of additive concentrations in the PVC matrix. 
a
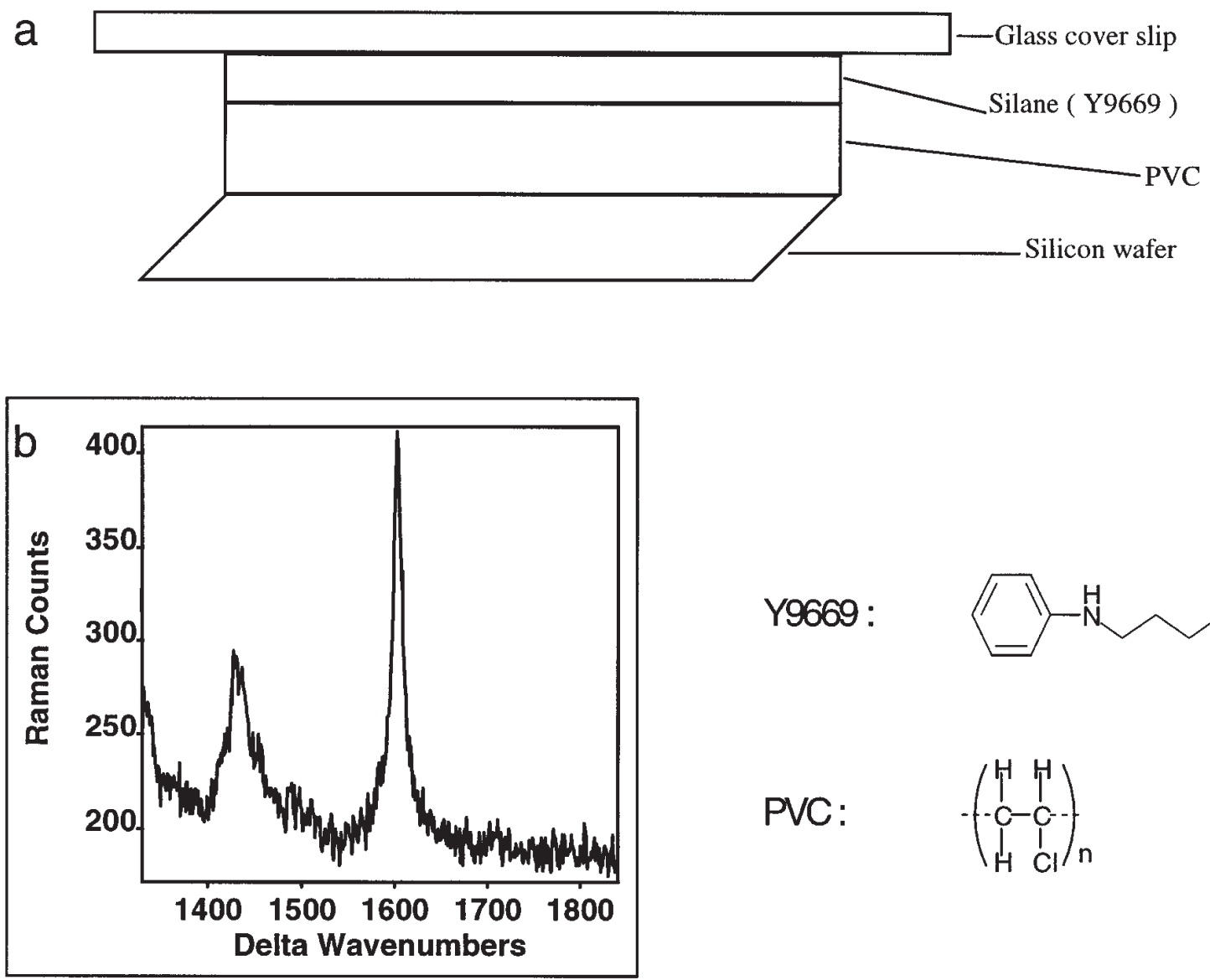

Y9669:<smiles>CO[Si](CCCNc1ccccc1)(OC)OC</smiles>

PVC:

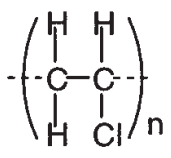

C

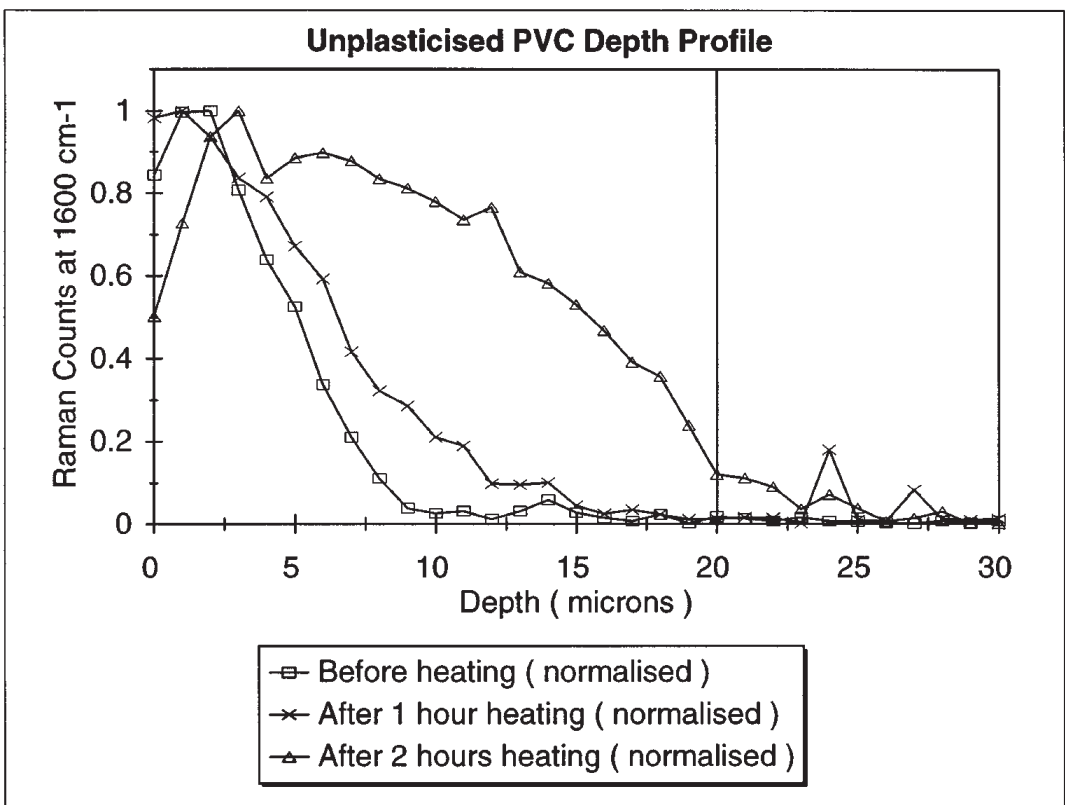

Figure 4. (a) The silane/PVC matrix structure; (b) the $1602 \mathrm{~cm}^{-1}$ band of Y9669; (c) The silane distribution with depth into PVC as a function of annealing temperature. 
by measuring the area under the phenyl ring vibrational band at $\sim 1600 \mathrm{~cm}^{-1}$ (Fig. 4b). In figure 4c, we demonstrate how we have employed CRM to measure the depth profile of Y9669 (initially brushed onto the surface of a $20 \mu \mathrm{m}$ pure PVC film) as a function of the annealing process, at $70^{\circ} \mathrm{C}$. At longer annealing times the diffusion of silane towards the interface is clearly enhanced, correlating well with commercial conditions for laminate production (which involves formulating the polymer with silane and then heating between glass sheets).

\section{Acknowledgments}

We acknowledge with gratitude the support of EPSRC, Zeneca plc, ICI (Wilton) and Pilkington, and valuable discussions with Ron Swart, David Hodge, Neil Everall and Paul Holmes.

\section{References}

1. Tabaksblat, R.; Meier, R.J.; Kip, B.J. Applied Spectrosc. 1992, 46,60

2. Williams, K.J.P.; Pitt, G.D.; Smith, J.E., Whitley, A.; Batcheldor, D.N.; Hayward, I.P. J. Raman Spectrosc. 1994, 25, 131.

3. Treado, P.J.; Morris, M.J. Applied Spectrosc. 1994, 29, 1-38.

4. Pittcon Report, Spectrosc. Europe, March-April, 1999, 30-31.

5. Adar, F.; Neother, H. Polymer 1985, 26, 1935.

6. Sammon, C. PhD Thesis, Sheffield Hallam University, 1997.

7. Mura, C.; Swart, R.M.; Hodge, D.J.; Yarwood, J. Polymer 1999 (in press).

8. Kasteleiner, T.; Swart, M.; Hodge, D.J.; Yarwood, J. J. Raman Spectrosc. 1996, 27, 695.

9. Eaton, P. PhD Thesis, Sheffield Hallam University, 1998; Eaton, P.; Holmes, P.; Yarwood, J. Applied Spectrosc. 1999 (submitted).

10. Chaudhury, M.K.; Gentle, T.M.; Plueddeman, E.P.; J. Adhes. Sci. Techn. 1990, 4, 597. 